\section{Fecundidade das mulheres autodeclaradas indígenas residentes em Minas Gerais, Brasil: uma análise a partir do Censo Demográfico 2000}

\author{
Fertility of indigenous women in Minas Gerais \\ State, Brazil: an analysis using the 2000 census
}

\begin{abstract}
Indigenous populations living in villages in Brazil have presented high total fertility rates (TFR) that have increased over time in some cases. Meanwhile, data from the Brazilian Institute of Geography and Statistics (IBGE) or National Census Bureau show a decline in the TFR for the total self-declared indigenous population (combining urban, rural, and specific rural residence). The current study uses data from the 2000 Population Census to describe and compare the structure and level of period fertility for the cohort of self-declared indigenous women living in the State of Minas Gerais. We calculated age-specific fertility rates (ASFR), the period TFR for 2000, and the cohort TFR for women 50 years and older. According to the findings, self-declared indigenous women living in Minas Gerais experienced high fertility in the past, regardless of their place of residence. In conclusion, the 2000 Population Census is an important data source for studies on indigenous populations in Brazil, based on the high coverage and wealth of information.
\end{abstract}

South American Indians; Demography; Fertility
Cláudio Santiago Dias Júnior 1,2 Ana Paula de Andrade Verona 1 João Luiz Pena ${ }^{2}$ George Luiz Lins Machado-Coelho ${ }^{3}$

\section{Introdução}

As investigações empíricas que têm a demografia indígena como objeto de estudo têm avançado muito nos últimos vinte anos 1. Esses avanços podem ser mensurados principalmente por meio do grande volume de produção científica que normalmente utiliza dados etnográficos e laudos antropológicos. É importante salientar que esses estudos muitas vezes abordam a população indígena residente na Amazônia, deixando em segundo plano as demais regiões do país. Por outro lado, estudos sobre a demografia indígena utilizando os censos demográficos do Instituto Brasileiro de Geografia e Estatística (IBGE; http://www.ibge.gov.br) ainda são incipientes, mesmo trazendo informações que permitem analisar o grupo autodeclarado indígena sob vários aspectos sócio-econômicos e demográficos.

Buscando reduzir essas lacunas, este trabalho utiliza dados do Censo Demográfico 2000 para descrever e comparar a estrutura e o nível da fecundidade de período e coorte das mulheres autodeclaradas indígenas residentes em Minas Gerais, Brasil, segundo a situação de domicílio. A escolha do Estado de Minas Gerais se justifica pelo número significativo de indígenas autodeclarados ali residentes, pela presença de povos indígenas aldeados e pelo limitado número de estudos demográficos sobre a população indígena deste estado. 
A comparação entre as medidas de fecundidade de período e coorte evidencia as mudanças no comportamento reprodutivo ao longo do tempo. Esta estratégia foi utilizada dada a falta de informações sobre populações indígenas no censo de 1970, que não possui o quesito cor/raça, e no de 1980, que por sua vez não possui a categoria indígena no quesito cor/raça. Embora o censo de 1991 possua a variável cor/raça incluindo a categoria indígena, ele não foi utilizado por dois motivos. O primeiro por considerar que a população autodeclarada indígena nesse censo é muito diferente da população captada pelo censo de 2000 2. Em Minas Gerais, por exemplo, a população autodeclarada indígena em 2000 é quase nove vezes maior; um crescimento pouco comum em termos demográficos que precisa urgentemente ser analisado. O segundo motivo se deve ao fato de alguns estudos enfatizarem que os dados do censo de 1991 não permitem boas estimativas da fecundidade para a população autodeclarada indígena 3 .

\section{População autodeclarada indígena e sua distribuição espacial}

No censo de 2000 , a população autodeclarada indígena foi identificada com base na pergunta sobre raça/cor, que inclui as categorias: branco, preto, pardo, amarelo e indígena. O número de indivíduos autodeclarados indígenas em Minas Gerais foi de 48.720 , sendo que $78 \%$ residiam em áreas urbanas e $22 \%$ nas áreas rurais. Do total da população indígena residente nas áreas rurais, 55\% estavam no chamado rural específico. Segundo o IBGE, o rural específico é definido como as áreas rurais dos municípios com Terras Indígenas 2. Do total da população, 23.508 eram homens e 25.212 mulheres, $32 \%$ estavam no grupo etário de $0-14$ anos, $62 \%$ pertenciam ao grupo etário de 15-64 anos, e 6\% tinham 65 anos e mais de idade. A população autodeclarada indígena em Minas Gerais é a quinta maior do Brasil, ficando atrás apenas das populações autodeclaradas indígenas residentes nos estados do Amazonas, Bahia, São Paulo e Mato Grosso do Sul, respectivamente.

A Figura 1 mostra a distribuição espacial (segundo município) da população autodeclarada indígena em Minas Gerais e a localização das Terras Indígenas presentes no estado, em 2000. Os municípios com suas respectivas Terras Indígenas são: Caldas/Xukuru-Karirí; Martinho Campos e Pompéu/Caxixó; Carmésia/Pataxó; Resplendor/Krenák; Santa Helena de Minas e Bertópolis/Maxakalí; São João das Missões/ Xakriabá; Coronel Murta/Pankararu e Aranã.
Esses municípios são aqueles com as bordas destacadas na Figura 1.

Dos 853 municípios mineiros, em 612 foram identificados até cinqüenta indivíduos que se autodeclararam indígenas, sendo que em 347 municípios nenhum indivíduo se autodeclarou como indígena. Além disso, em 125 municípios o total da população autodeclarada indígena variou entre 51 e 500 indivíduos. Apenas 16 municípios possuíam um contingente autodeclarado indígena superior a 500 indivíduos. Entre esses, as maiores concentrações foram observadas na Região Metropolitana de Belo Horizonte (com aproximadamente 15 mil autodeclarados indígenas) e nas grandes cidades pólo do Estado de Minas Gerais, como Uberlândia (1.551), Juiz de Fora (1.220), Montes Claros (1.153) e Governador Valadares (629). A exceção foi São João das Missões, que é o segundo município mineiro com o maior número de indivíduos autodeclarados indígenas (cerca de 4 mil). Cabe ressaltar que esse município abriga a Terra Indígena Xakriabá, a maior população indígena aldeada em Minas Gerais e uma das maiores do Brasil 4,5,6.

\section{Dados e métodos}

Este trabalho utilizou os dados de mulheres autodeclaradas indígenas com 15 anos e mais de idade, totalizando 19.792 indivíduos. Para mensurar a fecundidade dessas mulheres foram calculadas a taxa específica de fecundidade (TEF), a taxa de fecundidade total (TFT) de período para o ano 2000 e a de coorte, para as mulheres com 50 anos e mais de idade. O cálculo da TEF foi realizado da seguinte maneira:

$$
T E F_{x}=\frac{N_{x}}{M_{x}}(1),
$$

em que: $N_{x}=$ nascimentos no ano de referência para mulheres em idade ou grupo etário $x ; M_{x}=$ total de mulheres em idade ou grupo etário $x$ no meio do ano de referência.

Normalmente as TEFs são calculadas separadamente para cada grupo etário que vai de 1519 até 45-49 anos, isto é, o grupo de mulheres no período reprodutivo. Para calcular a TFT de período, somamos as TEFs e multiplicamos por cinco, que é o equivalente em anos de cada grupo etário. Para realizar esses cálculos foi utilizada a seguinte fórmula:

$$
T F T=\sum T E F * 5(2) .
$$

Para calcular a TFT de coorte foi utilizado um método demográfico de estimativa da fecundidade chamado razão de progressão por parturi- 

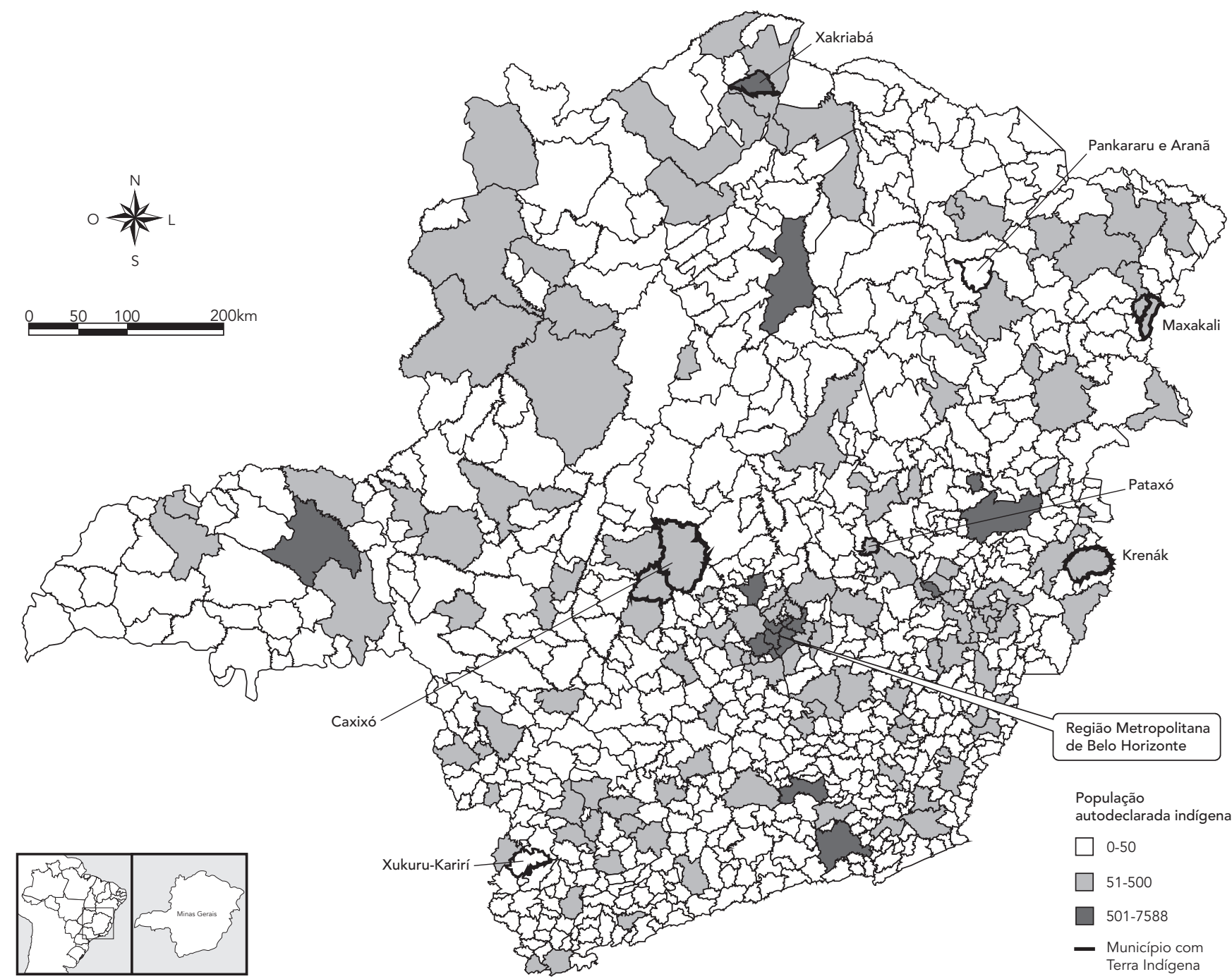

Fonte: Censo Demográfico 2000 (http://www.ibge.gov.br).

ção (RPP, parity progression ratio). Esse método estima a fecundidade e o seu padrão baseandose na informação de parturição de uma determinada coorte de mulheres (grupo de mulheres que pertencem ao mesmo intervalo etário). A RPP é a probabilidade de se ter um filho de ordem $x+1$, dado que se tenha um filho de ordem $x^{7}$. Geralmente, a RPP é utilizada em coortes que já encerraram a vida reprodutiva, isto é, coortes de mulheres com 50 anos e mais de idade. Portanto, uma característica dessa medida é que ela representa o comportamento reprodutivo passado da população, permitindo assim, fazer comparações com a fecundidade observada no período atual.

A fórmula (3) apresenta o cálculo da TFT a partir do somatório das RPPs:

$$
T F T=\frac{N_{x}}{M_{x}}=\frac{\sum_{x=1}^{n} N_{x}}{\sum_{x=0}^{n} M_{x}}=\sum_{x=1}^{n} \operatorname{RPP}(0, x)
$$

em que: $N_{x}=$ número de nascimentos de parturição $x ; M_{x}=$ número de mulheres com parturição $x$ (até parturição $=n$ ). 


\section{Resultados}

A Figura 2 apresenta os diferenciais de fecundidade (TFT) das mulheres autodeclaradas indígenas e das mulheres de Minas Gerais por situação de domicílio, e mostra as diferenças entre as TFTs de período e de coorte.

O primeiro aspecto a ser destacado na Figura 2 são os diferenciais da TFT de período por situação de domicílio em 2000. Nesse ano, a TFT de período da população indígena total era de 2,5 filhos por mulher em média, valor próximo ao encontrado para o Estado de Minas Gerais $(2,2)$. A baixa fecundidade observada para o total das mulheres autodeclaradas indígenas em 2000 se deve, em parte, ao fato desta população ser composta principalmente por mulheres residentes nas áreas urbanas (78\%), onde a TFT era de 2,1 filhos por mulher.

A TFT de período das mulheres autodeclaradas indígenas residentes nas áreas rurais em 2000 apresentam valores relativamente altos. Essas taxas indicam um comportamento reprodutivo distinto em relação às mulheres autodeclaradas indígenas que vivem nas áreas urbanas. Para as residentes nas áreas rurais, a TFT era de 4,3 filhos, enquanto que para as residentes no rural específico a TFT chegou a 5,3 filhos por mulher em média. Embora a TFT da área rural de Minas Gerais seja relativamente alta $(3,3)$, haja vista a TFT da área urbana do estado $(2,1)$, esta diferença é inferior à observada entre as mulheres autodeclaradas indígenas.

O segundo aspecto a ser enfatizado são os diferenciais da TFT de coorte por situação de domicílio. A Figura 2 deixa claro que independentemente da situação de domicílio, a TFT das mulheres autodeclaradas indígenas com 50 anos e mais são bastante altas. Além disso, os diferenciais de TFT por situação de domicílio são muito menores quando consideramos as medidas de coorte. Por exemplo, a diferença entre as TFTs de coorte das mulheres autodeclaradas indígenas residindo no rural específico e nas áreas urbanas é de 1,1 filho $(6,8-5,7)$, enquanto que a diferença entre as medidas de TFT de período entre as mulheres residindo nestas áreas é de 3,2 filhos $(5,3-2,1)$.

O último aspecto analisado com base na Figura 2 são os diferencias de TFT de período e coorte. A comparação entre eles permite uma análise temporal do comportamento da fecundidade. Ao confrontar as TFTs de período e coorte, por situação de domicílio, verifica-se que de uma maneira geral as mulheres autodeclaradas indígenas experimentaram o declínio da fecundidade. Esse declínio é mais rápido entre as residentes nas áreas urbanas, mas existiu também nas áreas rurais, inclusive naquelas que possuem Terras Indígenas. A diferença observada entre as TFTs de período e coorte nas áreas urbanas foi de 3,6 filhos $(5,7-2,1)$, ao passo que no rural específico esta diferença foi de $1,5(6,8-5,3)$. Essa comparação mostra que o processo de declínio da fecundidade entre as mulheres autodeclaradas indígenas em Minas Gerais ocorreu de forma bastante diferenciada por situação de domicílio. Fica claro que entre aquelas vivendo nas áreas urbanas esse processo foi muito mais rápido.

Figura 2

Taxa de fecundidade total de período e de coorte, segundo situação de domicílio. Minas Gerais, Brasil, 2000.

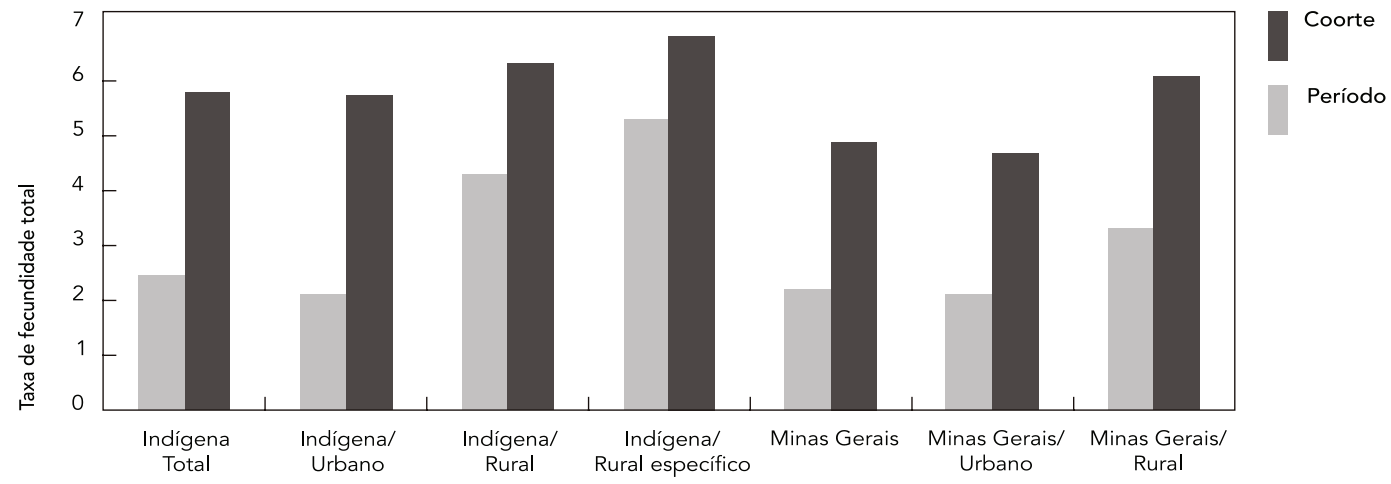

Fonte: Censo Demográfico 2000 (http://www.ibge.gov.br). 
A Figura 3 mostra o comportamento das TEFs das mulheres autodeclaradas indígenas, segundo a situação de domicílio, e de todas as mulheres residentes em Minas Gerais em 2000. As autodeclaradas indígenas que vivem nas áreas rural e rural específico apresentam estruturas etárias típicas de populações com fecundidade alta. Ambas possuem um padrão envelhecido, com o ápice da curva no grupo etário de 25-29 anos, e com fecundidade relativamente alta entre as idades de 30-39 anos.

Ao contrário, a população residente nas áreas urbanas apresenta uma estrutura de fecundidade mais "achatada", na qual os grupos de mulheres entre 20-24 e 25-29 anos possuem praticamente a mesma TEF. A forma dessa curva pode indicar um processo de rejuvenescimento da fecundidade nas áreas urbanas, que é reflexo do declínio da fecundidade (como mostrado na Figura 2). Esse rejuvenescimento implica uma redução da idade média de fecundidade. Os dados não permitem observar essa mudança ao longo do tempo, contudo, permitem calcular e comparar esta média segundo situação de domicílio. Entre as mulheres residentes nas áreas urbanas, a idade média de fecundidade é igual a 27,7 anos, e para aquelas que vivem no rural específico esta média é de 29,0. Existe, portanto, uma concentração maior da fecundidade nas idades mais jovens entre mulheres autodeclaradas indígenas residentes em áreas urbanas, em 2000.

A Figura 4 mostra a distribuição percentual da fecundidade, segundo o grupo etário e situa- ção de domicílio. Entre as mulheres autodeclaradas indígenas, o grupo de 25-29 anos é responsável pela maioria dos nascimentos, independente da situação de domicílio. Entre as mulheres indígenas residentes nas áreas rurais com Terras Indígenas, $73 \%$ dos nascimentos ocorrem entre aquelas com 25 anos e mais de idade. Tal observação é um indicativo de um comportamento reprodutivo ativo das mulheres indígenas nessas áreas até as idades mais avançadas. Ao contrário, na população total de Minas Gerais há uma concentração de $43 \%$ dos nascimentos entre as mulheres com até 24 anos.

Outro aspecto importante mostrado na Figura 4 é a baixa participação da fecundidade de adolescentes (15-19 anos) entre as mulheres autodeclaradas indígenas residentes no rural específico. Nos demais grupos, a participação da fecundidade das adolescentes é quase três vezes maior, o que representa cerca de $15 \%$ da fecundidade total destes grupos.

\section{Discussão}

De uma maneira geral, estudos de caso revelam altas taxas de fecundidade da população indígena, que em conjunto com o declínio das taxas de mortalidade infantil têm proporcionado elevadas taxas de crescimento anual 8,9. Esses achados vão de encontro com a teoria desenvolvida por Darcy Ribeiro nos anos 50, que previa uma redução progressiva da população autóctone, po-

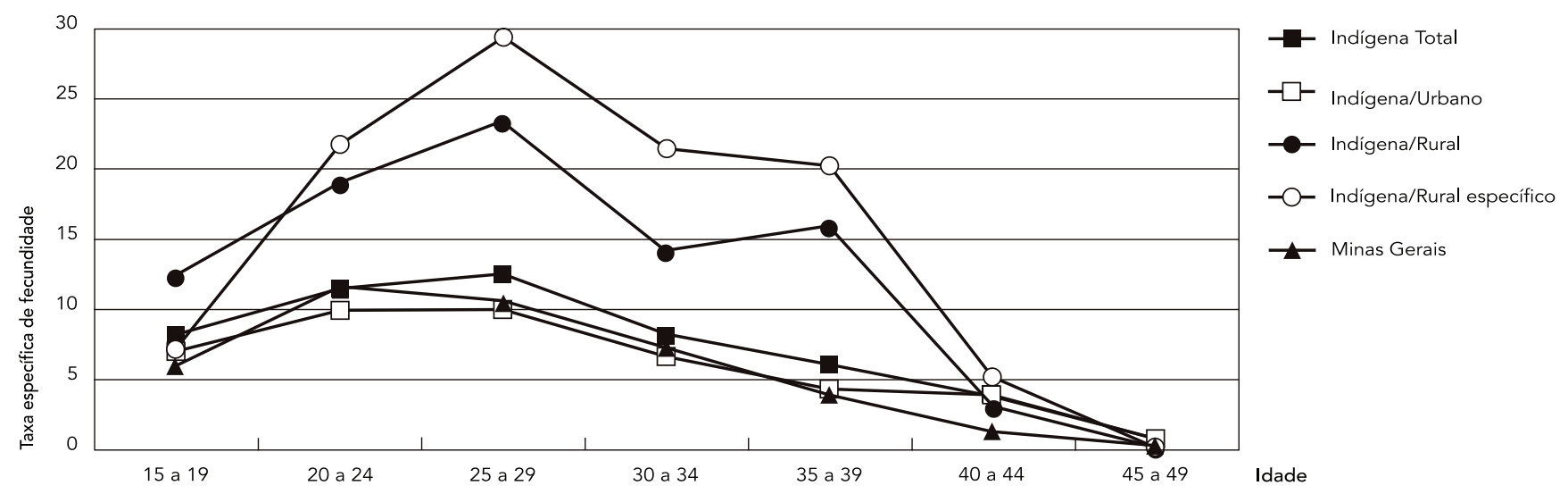

Fonte: Censo Demográfico 2000 (http://www.ibge.gov.br). 


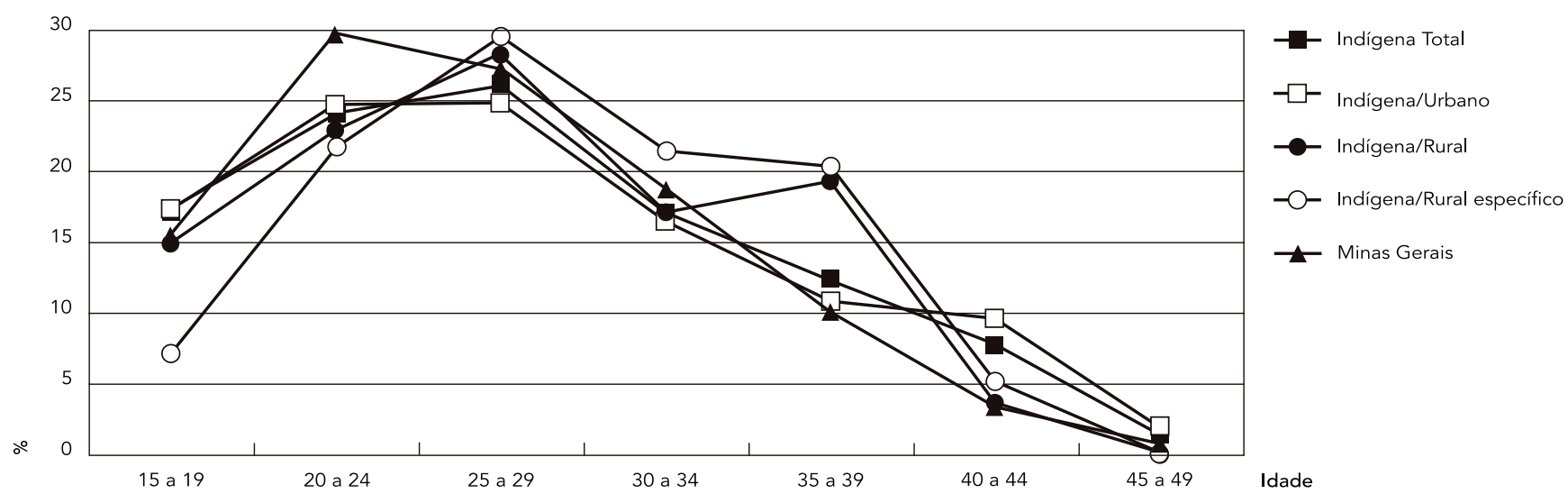

Fonte: Censo Demográfico 2000 (http://www.ibge.gov.br).

dendo chegar, no extremo, à extinção 10,11,12. Ao contrário da previsão de Darcy Ribeiro, segundo esses estudos de caso a população indígena tem se recuperado das graves crises populacionais associadas ao estabelecimento do contato com a sociedade ocidental 13,14. Por outro lado, em análises geradas com base nos censos demográficos de 1991 e 2000, observou-se um declínio da fecundidade da população autodeclarada indígena concomitante com altas taxas de crescimento $3,15,16$. Todas essas mudanças ocorrem em graus diferenciados, dependendo da situação de domicílio (urbano, rural, rural específico) 15.

Estudos de caso mostram que a fecundidade da população indígena aldeada está bem acima da média brasileira. Santos et al. 10, ao analisarem dados oriundos de diversos recenseamentos e história reprodutiva das mulheres Xavánte de Pimentel Barbosa, no Mato Grosso, construíram uma série histórica da TFT. No período de 1942-1956, a TFT era de 8,0 filhos por mulher, declinando para 5,9 nos anos entre 1957-1971. Em seguida, entre 1972-1990, observou-se uma recuperação da TFT, que chegou a 7,9 filhos por mulher. Segundo os autores, as mulheres Xavánte alegaram que no período de 1957-1971 houve um aumento da mortalidade infantil, fazendo com que elas perdessem a vontade de ter filhos, uma vez que não queriam ver a prole morrer. Em outro estudo, Souza \& Santos 17 observaram que entre os Xavánte de Sangradouro-Volta Grande, Mato Grosso, no período de 1993-1997, a TFT estava próxima de 8,6 filhos. Utilizando dados agregados retirados de diversas pesquisas realizadas entre os anos de 1980 e 2000, McSweeney \& Arps 9 encontraram uma TFT de 7,5 filhos para as mulheres indígenas no Brasil.

Recentemente, Campanário 18 utilizou o modelo de Evadan para corrigir os dados levantados desde 1965 pela Universidade Federal de São Paulo e encontrou uma TFT de 10,2 filhos por mulher, entre os índios Kayabí do Parque Indígena do Xingu, no período de 1995-2000 (o nível mais alto de fecundidade já identificado no Brasil). De acordo com o autor, nessa mesma etnia, no período de 1970-1975, a TFT era de 5,3 filhos por mulher. Outro estudo de caso, focalizando os Sateré-Mawé, no Estado do Amazonas nos anos de 2002-2003, Teixeira \& Brasil 19 estimaram uma TFT de 8,1 filhos por mulher. Já Pagliaro \& Junqueira 14 encontraram entre os Kamayurá uma TFT de 6,2 filhos no período de 2000-2003.

Ao contrário dos resultados observados nesses estudos de caso, análises baseadas nos censos demográficos apontam para uma redução da fecundidade entre as mulheres autodeclaradas indígenas no Brasil 15. Segundo Wong et al. 15, o declínio da TFT entre as mulheres autodeclaradas indígenas observado no período entre 1991-2000 pode ser explicado, em grande parte, pelo aumento do peso relativo das residentes nas áreas urbanas. Em 1991, as autodeclaradas indígenas urbanas eram $24 \%$ do total, e em 2000 passaram a representar $52 \% 2$. Os autores ainda mostram que no ano 2000 a TFT das áreas rurais permaneceram altas, sendo que a TFT do rural 
específico é superior a 6,0 filhos por mulher. Sendo essas áreas as regiões onde se encontram as Terras Indígenas, podemos tomar esse valor como sendo a TFT dos povos indígenas aldeados. Cabe destacar que esse valor é compatível com os valores observados pelos estudos de caso realizados em diversas Terras Indígenas 15.

Este trabalho mostra que o nível da fecundidade das autodeclaradas indígenas em Minas Gerais, em 2000, difere por situação de domicílio e por medidas de período e coorte. Mulheres residentes em áreas urbanas apresentam uma TFT bem inferior em relação àquelas que vivem nas áreas rural e rural específico. Esse resultado é semelhante ao encontrado para o Brasil 15. Contudo, é importante salientar que em Minas Gerais a porcentagem da população autodeclarada indígena vivendo em áreas urbanas é bastante superior àquela observada no Brasil. Em Minas Gerais esse valor é de 78\%, enquanto que no Brasil como um todo, $52 \%$ dos indivíduos autodeclarados indígenas viviam em áreas urbanas em 2000 2,15. O alto grau de urbanização em conjunto com uma TFT no nível da reposição populacional (2,1 filhos por mulher) pode colaborar para uma quase estabilização do tamanho da população autodeclarada indígena em Minas Gerais. No futuro, caso o grau de urbanização dessa população aumente (o que é esperado) e a fecundidade siga a tendência estadual, que é de declínio abaixo do nível de reposição (menos de 2,1 filhos por mulher), é esperada uma redução da população total autodeclarada indígena em Minas Gerais. Entretanto, essa tendência pode ser revertida caso se observe ondas significativas de migração de autodeclarados indígenas para o estado, e/ou aumente a autodeclaração indígena no próximo censo demográfico.

Além disso, os resultados deste trabalho deixam claro que as mulheres autodeclaradas indígenas residentes em Minas Gerais apresentam um passado de alta fecundidade, independente da situação de domicílio. Essa constatação se deu a partir da análise da TFT da coorte de mulheres com 50 anos e mais de idade. Comparando-se essa medida com a de período, percebe-se o declínio da fecundidade entre as autodeclaradas indígenas, independente da situação de domicílio. Contudo, essa comparação revela um substancial declínio da fecundidade das mulheres residentes nas áreas urbanas e um declínio moderado entre as residentes nas áreas rurais. Apesar desses achados, é importante salientar que os dados não permitem datar o início desse declínio.

A comparação entre as TFTs de período e coorte deve ser feita levando-se em consideração as características de cada medida. A primeira, a TFT de período, está sujeita a distorções devido a variações na idade média ao ter o filho. Por exemplo, a TFT pode ficar subestimada caso ocorra um aumento da idade média de fecundidade no período analisado, uma vez que este aumento pode indicar uma postergação da fecundidade e não uma diminuição desta. Essa distorção é chamada de efeito tempo 20.

Já a TFT de coorte representa a fecundidade real das mulheres com o ciclo reprodutivo encerrado (50 anos e mais). Contudo, essa medida também pode apresentar distorções caso a mortalidade de mulheres com filhos seja diferente da mortalidade de mulheres sem filhos. Se, por exemplo, a mortalidade de mulheres com filhos é maior do que daquelas sem filhos, a contribuição das primeiras para o cálculo da TFT pode ser menor do que seria caso não houvesse diferencias de mortalidade, causando assim uma subestimação na TFT de coorte. Apesar das possíveis distorções nas medidas de período e coorte, é esperado que elas sejam pequenas, não comprometendo a comparação entre o passado (coorte) e o presente (período) da fecundidade da população autodeclarada indígena.

Os resultados de Minas Gerais ainda revelam que a estrutura da fecundidade das autodeclaradas indígenas do estado também difere por situação de domicílio. Nas áreas rural e rural específico esta estrutura apresenta um padrão tardio, característico de populações com alta fecundidade. É muito provável que a proximidade dos valores culturais tradicionais e/ou a falta de informação e acesso a métodos contraceptivos modernos possam favorecer a alta fecundidade das mulheres autodeclaradas indígenas residentes nessas áreas. Esse mesmo tipo de padrão envelhecido também foi observado nas áreas rural e rural específico do Brasil 15. Já entre as autodeclaradas indígenas residentes nas áreas urbanas de Minas Gerais, a estrutura da fecundidade é mais "achatada", indicando uma concentração maior da fecundidade nas idades mais jovens.

Cabe salientar que nas análises demográficas o início do período reprodutivo feminino é quase sempre definido aos 15 anos de idade 7 . Contudo, alguns estudos demográficos de povos indígenas aldeados consideram o início da fecundidade em idades anteriores a essa ${ }^{14}$. Para esses casos, a utilização de idades abaixo de 15 anos pode ser adequada porque o denominador da TEF (número de mulheres no grupo etário) é geralmente pequeno, fazendo com que a ocorrência de poucos eventos (no caso, nascimentos) tenham um peso significativo no cálculo da TEF, e assim, na TFT. No caso deste trabalho, essa questão não se aplica, pois o número de mulheres de 10-14 anos é relativamente grande e o de nascimentos é muito pouco expressivo. Caso se optasse por incluir 
essas mulheres nos cálculos da fecundidade, as TFTs ficariam praticamente as mesmas, mesmo no caso do rural específico, que é uma proxy das populações indígenas aldeadas. No Censo Demográfico 2000, por exemplo, nenhuma mulher autodeclarada indígena entre 10-14 anos residente no rural específico de Minas Gerais teve filhos nos 12 meses anteriores ao censo.

Finalmente, é importante mencionar que os dados de Minas Gerais não permitem verificar diretamente o comportamento reprodutivo das mulheres indígenas aldeadas, e por conseqüência, por etnia. Eles permitem apenas sugerir que o comportamento observado entre as mulheres indígenas residentes nas áreas rurais com Terras Indígenas seja uma aproximação do comportamento das mulheres aldeadas. De qualquer forma, pode-se inferir que, apesar de alta, a fecundidade nas Terras Indígenas de Minas Gerais já começou a experimentar um processo de declínio.

\section{Conclusão}

Este estudo mostrou como se comporta a fecundidade das mulheres autodeclaradas indígenas residentes em Minas Gerais no ano 2000. Existem diferenças expressivas na TFT de período quando se considera a situação domiciliar da mulher. Por outro lado, ao comparar as medidas de período e coorte, foi observado o declínio da fecundidade das mulheres autodeclaradas indígenas, independente da situação de domicílio.

A utilização da medida de coorte é um avanço para a análise do comportamento reprodutivo da população autodeclarada indígena, uma vez que os censos demográficos de 1970 e 1980 não permitem calcular a fecundidade para este grupo populacional. Outra contribuição deste artigo para a literatura existente é a utilização dos dados de Minas Gerais: um estado fora da Região Amazônica, cuja população indígena ainda é pouco analisada.

Os resultados apresentados neste artigo deixam claro que o uso do Censo Demográfico 2000 é uma importante fonte de dados para os estudos sobre populações indígenas no Brasil, tanto pelo seu alto grau de cobertura quanto pela riqueza das informações coletadas. Não obstante, deve ser ressaltado que os quesitos para captar o "indígena” podem ser aperfeiçoados, incluindo, por exemplo, critérios como idioma ou língua falada e etnia a que pertence.

Além disso, a Fundação Nacional do Índio (FUNAI) e o IBGE deveriam envidar esforços e conhecimentos técnicos acumulados ao longo dos anos e realizar o censo nacional da população indígena, contando com a participação das próprias comunidades e lideranças indígenas, assim como foi feito pela Dirección General de Estadística, Encuestas y Censos (DGEEC; http:// www.dgeec.gov.py, acessado em 16/Fev/2008) em 2002, no Paraguai. 


\section{Resumo}

As populações indígenas aldeadas no Brasil apresentam alta taxa de fecundidade total (TFT), sendo que em alguns casos é observado o crescimento destas taxas ao longo do tempo. Já os dados do Instituto Brasileiro de Geografia e Estatística (IBGE) revelam um declínio da TFT da população autodeclarada indígena, independente da situação de domicílio (urbano, rural e rural específico). Este trabalho utiliza dados do Censo Demográfico 2000 para descrever e comparar a estrutura e o nível da fecundidade de período e coorte das mulheres autodeclaradas indígenas residentes em Minas Gerais, Brasil. Foram calculadas as taxas específicas de fecundidade (TEF), a TFT de período para o ano 2000 e a TFT de coorte para mulheres com cinqüenta anos e mais de idade. Os resultados mostram que as mulheres autodeclaradas indígenas residentes em Minas Gerais experimentaram um passado de alta fecundidade, independente da situação de domicílio. Concluiu-se que o uso do Censo Demográfico 2000 é uma importante fonte de dados para os estudos sobre populações indígenas no Brasil, tanto pelo seu alto grau de cobertura quanto pela riqueza das informações coletadas.

Índios Sul-Americanos; Demografia; Fecundidade

\section{Colaboradores}

C. S. Dias Júnior e A. P. A. Verona idealizaram o artigo, participaram da revisão bibliográfica, processamento $\mathrm{e}$ análise dos dados, análise dos resultados, e foram responsáveis pela redação final do artigo. J. L. Pena contribuiu na revisão bibliográfica e na revisão final do manuscrito. G. L. L. Machado-Coelho colaborou na revisão final do trabalho.

\section{Agradecimentos}

Ao Conselho Nacional de Desenvolvimento Científico e Tecnológico (CNPq, processo 401187/2005-5), à Fundação de Amparo à Pesquisa do Estado de Minas Gerais (FAPEMIG, processo APQ -3200-4.01/07), ao Ministério da Saúde (MS/SCTIE/DECIT-OPAS, processo Proj-505169210917) pelo financiamento parcial do trabalho.

\section{Referências}

1. Coimbra Jr. CEA, Garnelo L. Questões de saúde reprodutiva da mulher indígena no Brasil. Porto Velho: Universidade Federal de Rondônia; 2003. (Documento de Trabalho, 7).

2. Instituto Brasileiro de Geografia e Estatística. Tendências demográficas: uma análise dos indígenas com base nos resultados da amostra dos censos demográficos 1991 e 2000. Rio de Janeiro: Instituto Brasileiro de Geografia e Estatística; 2005.

3. Pereira NOM, Santos RV, Azevedo MM. Perfil demográfico e socioeconômico das pessoas que se autodeclararam indígenas nos censos demográficos de 1991 e 2000. In: Pagliaro H, Azevedo MM, Santos RV, organizadores. Demografia dos povos indígenas no Brasil. Rio de Janeiro: Editora Fiocruz; 2005. p. 155-66.

4. Pena JL. Perfil sanitário, indicadores demográficos e saúde ambiental após a implantação do distrito sanitário especial indígena: o caso dos Xakriabá em Minas Gerais [Dissertação de Mestrado]. Belo Horizonte: Programa de Pós-graduação em Saneamento, Meio-Ambiente e Recursos Hídricos, Universidade Federal de Minas Gerais; 2004.
5. Campos MB, Campos TB, Monte-Mór RLM. A questão da demografia indígena: o caso dos Xakriabá, no norte de Minas. In: XV Encontro Nacional de Estudos Populacionais, Associação Brasileira de Estudos Populacionais. http://www. abep.nepo.unicamp.br/encontro2006/docspdf/ ABEP2006_191.pdf (acessado em 16/Fev/2008).

6. Paraíso MHB. Os botocudos e sua trajetória histórica. In: Cunha MC, organizadora. História dos índios no Brasil: São Paulo: Editora Companhia das Letras; 1992. p. 413-30.

7. Preston SH, Heuveline P, Guillot M. Demography: measuring and modeling population processes. Malden: Blackwell; 2001.

8. Pagliaro H, Carvalho NS, Rodrigues D, Baruzzi RG. Demography dynamics of the Suyá, a Jê people of the Xingu Indigenous Park, Central Brazil, 19702004. Cad Saúde Pública 2007; 23:1071-81.

9. McSweeney K, Arps SA. Demographic turnaround: the rapid growth of indigenous populations in lowland Latin America. Lat Am Res Rev 2005; 40:3-29. 
10. Santos RV, Flowers NM, Coimbra Jr. CEA. Demografia, epidemias e organização social: os Xavánte de Pimentel Barbosa (Etéñitépa), Mato Grosso. In: Pagliaro H, Azevedo MM, Santos RV, organizadores. Demografia dos povos indígenas no Brasil. Rio de Janeiro: Editora Fiocruz; 2005. p. 59-78.

11. Ribeiro D. Culturas e línguas indígenas no Brasil. Educação e Ciências Sociais 1957; 2:5-102.

12. Ribeiro D. Convívio e contaminação: efeitos dissociativos da população provocados por epidemias em grupos indígenas. Sociologia 1956; 18:3-50.

13. Flowers NM. Crise e recuperação demográfica: os Xavánte de Pimentel Barbosa, Mato Grosso. In: Santos R, Coimbra Jr. CEA, organizadores. Saúde e povos indígenas. Rio de Janeiro: Editora Fiocruz; 1994. p. 213-42.

14. Pagliaro H, Junqueira C. Recuperação populacional e fecundidade dos Kamaiurá, povo Tupi do Alto Xingu, Brasil Central, 1970-2003. Saúde Soc 2007; 16:37-47.

15. Wong L, Morell MGG, Carvalho RL. Notas sobre o comportamento reprodutivo dos povos indígenas no período de 1991-2000. In: XV Encontro Nacional de Estudos Populacionais, Associação Brasileira de Estudos Populacionais. http://www. abep.nepo.unicamp.br/encontro2006/docspdf/ ABEP2006_538.pdf (acessado em 18/Fev/2008).
16. Pagliario H, Azevedo MM, Santos RV. Demografia dos povos indígenas no Brasil: um panorama crítico. In: Pagliaro H, Azevedo MM, Santos RV, organizadores. Demografia dos povos indígenas no Brasil. Rio de Janeiro: Editora Fiocruz; 2005. p. 11-32.

17. Souza LG, Santos RV. Perfil demográfico da população indígena Xavánte de Sangradouro-Volta Grande, Mato Grosso (1993-1997), Brasil. Cad Saúde Pública 2001; 17:355-65.

18. Campanário P. Estimativas de fecundidade e de mortalidade de populações de pequena escala através de um modelo demográfico. In: Pagliaro $\mathrm{H}$, Azevedo MM, Santos RV, organizadores. Demografia dos povos indígenas no Brasil. Rio de Janeiro: Editora Fiocruz; 2005. p. 103-18.

19. Teixeira P, Brasil MC. Estudo demográfico dos Sateré-Mawé: um exemplo de censo participativo. In: Pagliaro H, Azevedo MM, Santos RV, organizadores. Demografia dos povos indígenas no Brasil. Rio de Janeiro: Editora Fiocruz; 2005. p. 135-54.

20. Bongaarts J, Feeney G. On the quantum and tempo of fertility. Popul Dev Rev 1998; 24:271-91.

Recebido em 21/Nov/2007

Versão final reapresentada em 26/Fev/2008

Aprovado em 31/Mar/2008 